\title{
Acceptance of the bodypainting as supportive method to learn the surface locomotor apparatus anatomy of the horse
}

\author{
R. Senos ${ }^{1}{ }^{2}$, M.S. Ribeiro ${ }^{1}$, K. de Souza Martins ${ }^{1}$, L.V. Pereira ${ }^{1}$, M.F. Mattos ${ }^{1}$, \\ J.R. Kfoury Júnior ${ }^{2}$, M.R. Rodrigues ${ }^{1}$ \\ ${ }^{1}$ Department of Morphology, Universidade Federal Fluminense, Brazil \\ ${ }^{2}$ Anatomy Section, Department of Surgery, Universidade de São Paulo, Brazil
}

[Received 7 January 2015; Accepted 26 February 2015]

\begin{abstract}
Although bodypainting has been reported as a great resource for teaching surface anatomy of humans, its use in veterinary anatomy has not been scientifically reported. In the present study, bodypainting was performed on 4 horses for anatomy teaching purposes of the equine locomotor apparatus. We aimed to use the bodypainting method as an additional tool to classic teaching and to test the relevance of our purpose. Twenty one Brazilian veterinary students were given a 90-min session, which included a presentation of painted horses, with opportunities for the students to ask questions and to palpate anatomic locations on the horses. Based on a questionnaire, there was unanimous student satisfaction with this technique. Furthermore, student scores on practical tests to evaluate the attention retain given immediately before and $1 \mathrm{~h}$ after the session were $33.9 \pm 19.8 \%$ and $69.0 \pm 18.4 \%$, respectively $(p<0.001)$. We concluded that bodypainting has great potential for support the classic lectures of the equine locomotor apparatus. (Folia Morphol 2015; 74, 4: 503-507)
\end{abstract}

Key words: body painting, bone, education, equine, medicine, muscle, veterinary anatomy

\section{INTRODUCTION}

Veterinary Anatomy is one of the most challenged subjects by undergraduate students and professors at the Universidade Federal Fluminense (Niterói, RJ, Brazil) that do not believe classic lectures are really efficient in veterinary medical curricula nowadays. In the last 50 years, the way of teaching has not improved so much, besides the addition of lecture presentation resources like the use of multimedia projectors. The classical approach solely to teaching the locomotor apparatus of the domestic animals, including lectures and presentation of cadavers, resulted in decreasing student attendance over time $[8,10,11]$. Students usually complain against monotony, passive posture, formaldehyde unpleasant effects and how descriptive lessons would help them in clinical practices.

Various alternative teaching and learning methods have been implemented to improve knowledge and interest of both human and veterinary anatomy in last decades, including videos, plastination, 3-dimensional computed tomography images, and problem-based learning $[6,12,14]$. Notwithstanding, the application of novel techniques for teaching veterinary anatomy in live animals is still rare [2]. 

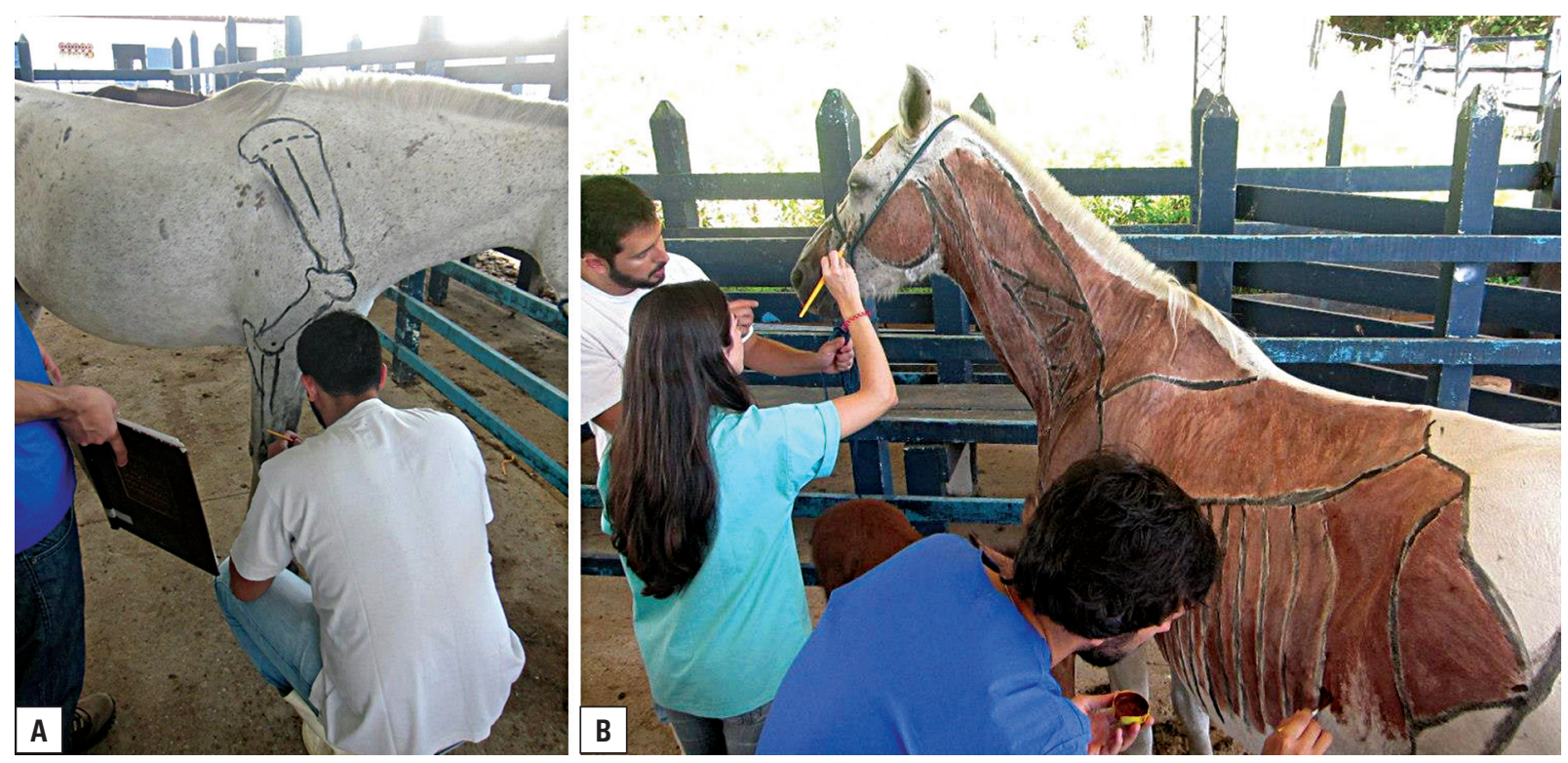

Figure 1. Drawing the anatomy on the skin of the horses; A. Bones; B. Muscles.

The knowledge of the position, size and shape of internal and surface structures in living specimens is the basic condition for good quality of physical examination and clinical practice [9]. However, in veterinary medicine classic courses it is not rare finding students who reach the clinical subjects without these principles consolidated.

Bodypainting for anatomical purposes was apparently first described by Op Den Akker [9] in human anatomy as painting internal structures on the surface of the body. The authors were inspired by the use of the bodypainting technique in dramatic arts and glossy magazines, and decided to apply this technique of bodypainting to teach surface anatomy. Although there have been several subsequent reports in humans $[1,3,4,7]$, there is apparently a paucity of reports regarding the application of this technique to teach veterinary anatomy.

The objective of the present study was to obtain a short acceptance evaluation of the bodypainting technique by students in aid to classic educational methods for teaching the equine locomotor apparatus to undergraduate veterinary students.

\section{MATERIALS AND METHODS}

\section{Animal use and ethical principles}

The protocol of the practice was approved and certified under register number 493 by the Bioethics Commission of the Universidade Federal Fluminense in agreement to ethical principles of animal use for academic purposes.

\section{The practice}

Twenty one first-year students of the Veterinary Anatomy subject of the Universidade Federal Fluminense in Brazil volunteered to participate in this study. They had $220 \mathrm{~h}$ of conventional presentations ( $60 \mathrm{~h}$ of theoretical and $160 \mathrm{~h}$ of laboratories over 20 weeks) in anatomy of the locomotor apparatus of the domestic animals prior to the bodypainting session. One week after the end of the regular semester of classic lessons, we conducted the proposed method.

Four horses were used; 2 of them had the anatomy of the bones and muscles painted on the skin (Fig. 1), whereas the other 2 had crosses painted on the skin in parts of the body where students were expected to recognise bone and muscle structures during the attention tests. Painting was done by professors and assistants using gouache ink (Acrilex company, Sao Bernardo do Campo city, Sao Paulo State, Brazil) away from the students presence. The teaching session lasted $90 \mathrm{~min}$, including anatomical explanations, and opportunities for students to palpate the horses and ask questions. The Nomina Anatomica Veterinaria [13] was applied.

After the session, students had 15 min to take pictures of the animals and at the end horses were washed with water to remove the ink and relax. 


\section{Qualitative analysis of the feedback}

After the experience, professors and assistants got together to report their impressions on the use the bodypainting and behaviour of the students. In addition, assistants got information regarding this experience repercussion in the faculty environment.

During and just after the session, we also recorded students' comments regarding that practice.

\section{Acceptance test}

Method acceptance was determined in anonymous multiple choices forms answered by students $1 \mathrm{~h}$ after presentation. There were four questions: 1) How do you evaluate the method? - possible choices were very good; good; regular, bad or very bad; 2) How useful was the method in the learning process? - possible answers were very useful, useful, regular, little useful or useless. Were you confident that you could identify anatomical structures in living horses; 3) Before the bodypainting presentation; 4) After the presentation - possible answers yes or no?

\section{Students' attention evaluation}

To assess the attention of the students on the method, two tests were applied. Students performed Test 1 before the bodypainting session, whereas Test 2 was taken $1 \mathrm{~h}$ after the session (Fig. 2). Random identification numbers were used to preserve student anonymity. Each test consisted of identifying and naming 8 randomly selected anatomical structures -4 bone and 4 muscle structures - in the surface of living horses including the head, neck, trunk and limbs (Table 1). Students were allowed to palpate the horses during the tests. The tests did not repeat the same structures.

\section{Statistical analysis}

The acceptance test was evaluated using percentages for each question. Paired Student's t-test was used to compare answers of the Questions 3 and 4.
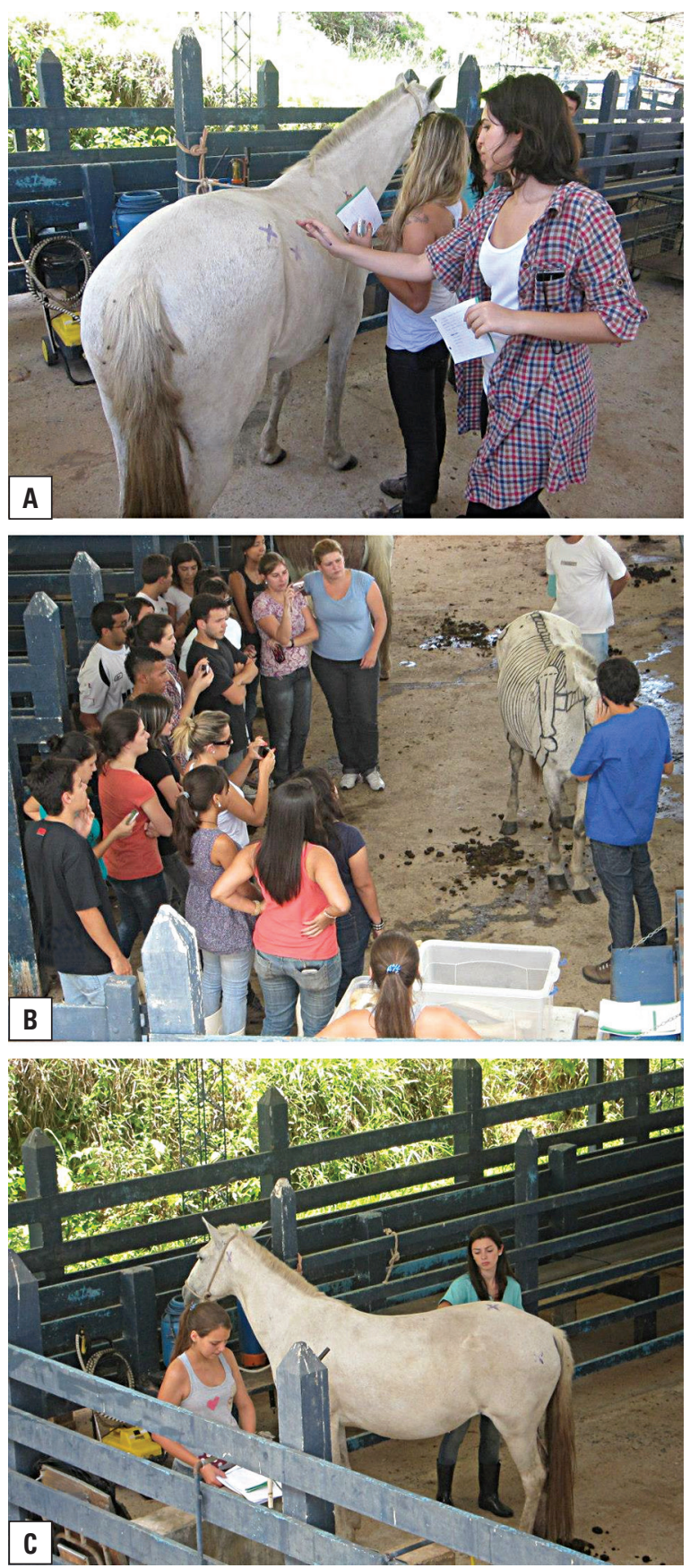

Figure 2. A. Test 1; B. Bodypainting presentation; C. Test 2.

Table 1. Structures that should be identified in test 1 and 2

\begin{tabular}{lcc}
\hline & Test $\mathbf{1}$ & Test $\mathbf{2}$ \\
\hline Bone structures & Ala atlantis (Atlas) & Epicondylus lateralis (Os humerus) \\
& Cartilago scapulae (Os scapula) & Processus styloideus lateralis (Os radius) \\
Corpus costae $(\# 18)$ & Tuber sacrale (Os ilium) \\
Muscle structures & Tuber coxae (Os ilium) & Tuber ischiadicum (Os ischii) \\
& M. masseter & M. brachiocephalicus \\
M. latissimus dorsi & M. serratus ventralis thoracis \\
M. deltoideus & M. gluteus superficialis \\
\hline
\end{tabular}


Table 2. Data obtained from grades of Test 1 and Test 2

\begin{tabular}{lcccc}
\hline & Average grade [\%] & Median & Highest grade [\%] & Lowest grade [\%] \\
\hline Test 1 & $33.93 \pm 19.82$ & 25.00 & 75.00 & 12.50 \\
Test 2 & $69.05 \pm 18.38$ & 62.50 & 100 & 37.50 \\
\hline
\end{tabular}

For the attention tests, the percentage of correct answers was used to establish grades; thereafter, mean, standard deviation and median of the grades were calculated. Furthermore, a paired Student's t-test was used to compare grades between Tests 1 and 2 [15].

All statistical analyses were done using Epilnfo TM version 7.1.3 software (Centres for Disease Control and Prevention - Atlanta, Georgia, USA).

\section{RESULTS}

Five topics emerged in our analysis after the bodypaiting session.

\section{Professor and assistants' observation}

The professors and assistants expressed positively the experience, because of the students' enthusiasm and extroversion. Although the euphoric atmosphere, the students adopted respectful behaviour on the animal use.

Students palpated the animals carefully avoiding the distress. The generosity with the animals could be observed in all their acts, including proactive interest at the end of the session in helping to give the horses the bath.

\section{Students' perception}

The students' comments recorded by the professors and assistants summarise the atmosphere after the bodypainting session.

"Leaving the classroom and labs to learn in the field is really stimulating. We should do it more often."

"I have never thought about learning anatomy in live animals. It seems more real and clinical. The colours and animal movements make easier to understand the role of the muscles and their regional relationship."

"Whenever visiting my parents' farm, I always looked at the horses and bovines but I could not match the anatomical structures learned in the lab to live animals. After this practice, I feel I can. I suggest we have this kind of practice each month because it helps learning."

\section{Social repercussion}

Days, weeks and months after this experience, the bodypainting session caught special attention at the faculty. Students that did not participated on the session asked about it to the volunteers, assistants and professors.

Besides, social networks revealed many publishing and topics using the pictures took by the students in the end of the session.

\section{Acceptance test}

The responses to the acceptance test questions were as follows: 1) All students designated that the method was very good; 2) All students stated the methods as very useful; and regarding whether they could identify anatomical structures of the locomotor apparatus in living horses; 3 ) $28.5 \%$ could do so before bodypainting; whereas 4) $100 \%$ could do so after the presentation ( $p<0.001)$.

\section{Attention retain}

The attention tests results revealed an average increase of 35\% from Test 1 to Test 2 (Table 2). This is significant different ( $p<0.001)$.

One student showed $25 \%$ decrease of performance from Test 1 to Test 2 .

\section{DISCUSSION}

Although the positive perception of the professors and assistants, unanimous opinion that the bodypainting cannot substitute the classic lessons was considered. The sense of depth in surface anatomy is a great limitation for the bodypaiting. The use of cadaver is still the best method to construct a 3-dimensional anatomy concept, as stated previously [8]; however, the bodypainting can be an interesting tool to be added in anatomy teaching, even to create positioning comprehension to not palpable internal organs.

Regarding the social repercussion of the method, it is believed it could be encouraged in institutions where the veterinary anatomy subject is contested or worn. It is important to find methods that stimulate the students and academic environment. 
Our findings pointed out that the method was well accepted by all students, because none registered less than maximum acceptance grades in Questions 1 and 2 . It is believed that these positive answers are related to the direct contact with live animals that stimulated students' clinical perspectives. These results were consistent with those on bodypainting in human anatomy $[3,8]$.

The answers obtained in Questions 3 and 4 of acceptance test indicated that bodypainting method promoted the confidence of the students on surface anatomy knowledge. Once the results revealed a high prevalence of students who felt capable to find anatomical structures in live animals after session; the bodypainting improved self-reliance and clinical skills that was observed in India [8].

The tests applied to evaluate the students' attention suggested that the method was effective in keeping the students focused in the lecture. Overall, there was a significant increase from the average grades in Test 1 before the experience to Test 2 after the experience. We are aware that the Test 2 was applied just $1 \mathrm{~h}$ after the bodypainting session and it reflected on Test 2 grade. In addition, we agreed that colourful visual images increase students' memories $[3,8]$, which also reflected in the grades. Nevertheless, we emphasize that we were not evaluating the knowledge gain or retain, but the attention students kept in that experience due to the importance of new methods to improve the students interest in veterinary anatomy.

\section{CONCLUSIONS}

Based on the reported experience, we concluded that bodypainting in horses has great potential for using in addition to classic methods to teach anatomy for undergraduate veterinary students. Although this study was limited in scope, the results were important and relevant because the method can stimulate and consequently promote the learning process. This should certainly be helpful in clinical veterinary medicine practice and should be encouraged by educators [5]. In addition, the qualitative analysis was considered very positive by students and faculties. We believe that efficiency tests and the use of other domestic species models is necessary to best understanding on how useful the bodypainting is in veterinary anatomy teaching.

\section{ACKNOWLEDGEMENTS}

We thank Prof. Dr John Kastelic from University of Calgary for revising this manuscript and the Dean of the Faculty of Veterinary Medicine of the Universidade Federal Fluminense, Prof. Dr Nadia Almosny for supporting the study.

\section{REFERENCES}

1. Bergman EM, Sieben JM, Smailbegovic I, de Bruin ABH, Scherpbier AJJA, van der Vleuten CPM (2013) Constructive, collaborative, contextual and self-directed learning in surface anatomy education. Anat Sci Edu, 6: 114-124.

2. Braid F, Williams SB, Weller R (2012) Design and validation of a novel learning tool, the "Anato-Rug", for teaching equine topographical anatomy. Anat Sci Edu, 5: 256-263.

3. Finn GM, McLachlan JC (2010) A qualitative study of students responses to body painting. Anat Sci Edu, 3: 33-38.

4. Finn GM, White PM, Abdelbagi I (2011) The impact of color and role on retention of knowledge: a body-painting study within undergraduate medicine. Anat Sci Edu, 4: 311-317.

5. Jason H (2007) Becoming a truly helpful teacher: considerably more challenging, potentially more fun, than merely doing business as usual. Adv Physiol Edu, 31: 312-317.

6. Latorre RM, García-Sanz MP, Moreno M, Hernandez F, Gil F, López O, Ayala MD, Henry RW (2007) How useful is plastination in learning anatomy? JVME, 34: 172-176.

7. McMenamin PG (2008) Body painting as a tool in clinical anatomy teaching. Anat Sci Edu, 1: 139-144.

8. Nanjundaiah K, Chowdapurkar S (2012) Body-painting: a tool which can be used to teach surface anatomy. J Clin Diag Res, 6: 1405-1408.

9. Op Den Akker JW, Bohnen A, Oudegeest WJ, Hillen B (2002) Giving color to a new curriculum: bodypaint as a tool in medical education. Clin Anat, 15: 356-362.

10. Ozkadif S, Eken E (2012) Modernization process in veterinary anatomy education. Energy Educ Sci Technol-PT B, 4: 957-962.

11. Plendl J, Bahramsoltani $M$, Gemeinhardt $O$, Hünigen $H$, Käßmeyer S, Janczyk P (2009) Active participation instead of passive behavior opens up new vistas in education of veterinary anatomy and histology. Anat Histol Embryol, 38: 355-360.

12. Theoret $\mathrm{CL}$, Carmel ÉN, Bernier S (2007) Why dissection videos should not replace cadaver prosections in the gross veterinary anatomy curriculum: results from a comparative study. JVME, 34: 151-156.

13. World Association of Veterinary Anatomists (2005) Nomina Anatomica Veterinaria, 5th ed. Hannover, Columbia, Gent, Sapporo: World Association of Veterinary Anatomists.

14. Yamada K, Taniura T, Tanabe S, Yamaguchi M, Azemoto S, Wisner ER (2007) The use of multi-detector row computed tomography (MDCT) as an alternative to specimen preparation for anatomical instruction. JVME, 34: 143-150.

15. Zar JH (1998) Bioestatistical analysis. 4th Ed. Prentice-Hall, Englewood Cliffs, New Jersey. 